\title{
Anderson localization for a supersymmetric sigma model
}

\author{
M. Disertori ${ }^{a *}$, T. Spencer ${ }^{b}$ \\ a) Laboratoire de Mathématiques Raphaël Salem, UMR CNRS 6085 \\ Université de Rouen, 76801, France \\ b) Institute for Advanced Study, Einstein Drive, \\ Princeton, NJ 08540, USA
}

October 7, 2018

\begin{abstract}
We study a lattice sigma model which is expected to reflect the Anderson localization and delocalization transition for real symmetric band matrices in 3D. In this statistical mechanics model, the field takes values in a supermanifold based on the hyperbolic plane. The existence of a diffusive phase in 3 dimensions was proved in 2 for low temperatures. Here we prove localization at high temperatures for any dimension $d \geq 1$. Our analysis uses Ward identities coming from internal supersymmetry.
\end{abstract}

\section{Introduction}

It is well known that the study of localization properties in a disordered material can be translated to the study of correlation functions in a lattice field theory, with an internal hyperbolic supersymmetry (SUSY), [9, 5, 6, 8]. In the physics literature one usually assumes the sigma model approximation, which is believed to capture the essential features of the energy correlations and transport properties of the underlying quantum system.

*e-mail: margherita.disertori@univ-rouen.fr 
The SUSY field theories which are equivalent to the Anderson tightbinding model and random band matrices are difficult to analyze with mathematical rigor in more than one dimension. In this context, Zirnbauer introduced a lattice field model which may be thought of as a simplified version of one of Efetov's nonlinear sigma models [10, 3]. In Zirnbauer's sigma model the field takes values in a target space $H^{(2 \mid 2)}$ which is a supermanifold extension of the hyperbolic plane. The model is expected to reflect the spectral properties of random band matrices, such as localization and diffusion, in any dimension. In [10] localization was established in one dimensional chain by analyzing the transfer matrix. We refer to [2] for a historical introduction and motivations.

More recently the existence of a 'diffusive' phase at low temperatures ( $\beta$ large) has been proved for the $H^{(2 \mid 2)}$ model in three or more dimensions, see [2]. For $\beta$ small, a localized phase was expected. However, unlike conventional statistical mechanics models, the $H^{(2 \mid 2)}$ model has a noncompact hyperbolic symmetry and so high temperature expansions cannot be done in the usual way. In fact, it is known that the bosonic hyperbolic sigma model in 3D has no localized phase because its effective action is convex for all $\beta>0$. On the other hand, numerical simulations [4] indicated that the SUSY hyperbolic sigma model has a phase transition for $\beta<\beta_{c} \simeq 0.038$.

In this paper we show that for any dimension $d>1$ the $H^{(2 \mid 2)}$ model exhibits localization for $\beta^{1 / 2} \ln \beta^{-1} \leq 1 /(2 d-1)$. Thus the sigma model approximation captures the physics of both localization and diffusion. Moreover, for a one dimensional chain we recover localization for all values of $\beta$. Localization is also expected in 2D (see [2] section 1.4 and 4.3), for all values of $\beta$ by both the renormalization group and by a simple saddle analysis. However, a rigorous proof is still missing for this case.

The techniques employed in this work to prove localization are quite different from the ones used in [2] to prove extended states. The two papers can be read independently. The only common point is the use of supersymmetry to prove some identities. In the present case supersymmetry is applied only to prove that the partition function is normalized to 1 . We refer to Section 4 and Appendix $\mathrm{C}$ in [2] for an introduction to supersymmetric Ward identities. 


\subsection{The model}

Let $\Lambda$ be a finite subset in $\mathbb{Z}^{d}$ and $t_{j}$ a real variable for each site $j \in \Lambda$. We will consider periodic or Neumann boundary conditions. Accordingly for any two points $x, y \in \lambda,|x-y|$ will denote the Euclidian distance on the lattice (Neumann bc) or the periodized lattice (periodic bc).

We introduce the probability measure

$$
d \mu_{\Lambda}^{\varepsilon}(t)=\prod_{j \in \Lambda} \frac{d t_{j}}{\sqrt{2 \pi}} e^{-F_{\Lambda}(\nabla t)} e^{-M_{\Lambda}^{\varepsilon}(t)} \times\left[\sqrt{\operatorname{det} D_{\Lambda}^{\varepsilon}(t)}\right]
$$

where $d t_{j}$ is the Lebesgue measure, $F$ is the kinetic part and $M$ is the mass term:

$$
\begin{aligned}
F_{\Lambda}(\nabla t) & =\beta \sum_{\left(j j^{\prime}\right) \in \Lambda}\left(\cosh \left(t_{j}-t_{j^{\prime}}\right)-1\right) \\
M_{\Lambda}^{\varepsilon}(t) & =\sum_{j \in \Lambda} \varepsilon_{j}\left(\cosh t_{j}-1\right) .
\end{aligned}
$$

We denoted by $\left(j j^{\prime}\right)$ the nearest neighbor pairs $\left|j-j^{\prime}\right|=1 . D_{\Lambda}^{\varepsilon}(t)$ is a positive definite matrix defined by

$$
\begin{array}{cl}
\left(D_{\Lambda}^{\varepsilon}\right)_{i j}=0 & |i-j|>1 \\
\left(D_{\Lambda}^{\varepsilon}\right)_{i j}=-\beta & |i-j|=1 \\
\left(D_{\Lambda}^{\varepsilon}\right)_{j j}=\beta\left[2 d+V_{j}\right]+\varepsilon_{j} e^{-t_{j}} & i=j \\
& V_{j}=\sum_{k,(j k)}\left[e^{t_{k}-t_{j}}-1\right]
\end{array}
$$

and $\varepsilon_{j} \geq 0$ are regularizing parameters that are necessary to make the integral well defined. We remark that $V_{j}+2 d>0$ for all $t$ configuration and all $j$. Finally $\beta>0$ is a parameter that can be interpreted as a measure of the temperature or the disorder.

Note that the definition of the matrix $D$ differs from the one introduced in [2] eq. (1.1). It is not difficult to see that when you mix the term $\sum_{k} t_{k}$ and the determinant in the effective action (1.2) of [2] the result is indeed the determinant of the matrix $D$ above, more precisely

$$
A_{i j}=e^{t_{i}} D_{i j} e^{t_{j}}= \begin{cases}0 & |i-j|>1 \\ -\beta e^{t_{i}+t_{j}} & |i-j|=1 \\ \beta \sum_{i^{\prime},\left(i i^{\prime}\right)} e^{t_{i}+t_{i^{\prime}}} & i=j\end{cases}
$$


where $A$ is the matrix introduced in [2] eq. (1.1). For technical reasons the above representation is more convenient when we want to prove diffusion as in [2] while the other is more practical when we study localization (except in the proof of Theorem 2 where we will go back to the "diffusion" representation for a while).

Now, for any function $f(t)$ we will define its average by

$$
\langle f(t)\rangle=\int d \mu_{\Lambda}^{\varepsilon}(t) f(t) .
$$

Normalization and choice of $\varepsilon_{j}$ By internal supersymmetry (see [2] Sect. 4 and eq.(5.1)) this measure is already normalized to 1 so the partition function is

$$
Z_{\Lambda}^{\varepsilon}=\int d \mu_{\Lambda}^{\varepsilon}(t)=1
$$

This identity is true regardless of the boundary conditions and the values of $\beta$ or $\varepsilon_{j}$ as long as the integral is well defined. Since we consider $\beta>0$ fixed we only need $\varepsilon_{j}$ to be non zero at one lattice point. In the following we will consider three cases.

1. Uniform pinning: $\varepsilon_{j}=\varepsilon \leq \frac{1}{\Lambda}$ for all $j \in \Lambda$. The measure is translation invariant with periodic bc. The correlation function in this case has a divergent prefactor $1 / \varepsilon$ in the localized regime.

2. Two pinnings: $\varepsilon_{x}=\varepsilon_{y}=O(1)$ and $\varepsilon_{j}=0$ for all other points. This is the analog of inserting two electrical contacts in a metal sample.

3. One pinning at $j=0: \varepsilon_{0}=O(1)$ and $\varepsilon_{j}=0$ for all other points. This is more suitable for an interpretation of the model as a random walk in a random environment. Our results suggest that edge reinforced random walk (see [7]) will also localize when the reinforcement is strong.

The observable. We will study the correlation function

$$
G_{x y}:=D_{x y}^{-1}
$$

where $x, y$ can be any two points on the lattice such that both $\varepsilon_{x}>0$ and $\varepsilon_{y}>0$. This observable does not give information on localization properties in the case of one pinning point. In such case a good observable to study is

$$
\mathcal{O}_{j}=e^{+t_{j} / 2}
$$


where $j$ is any point in the lattice. This observable is analogous to $x_{e}^{1 / 4}$ in the notation of [7] where $e$ is an an edge $\left(j, j^{\prime}\right)$.

\subsection{Main results}

Theorem 1 Let $\varepsilon_{x}>0, \varepsilon_{y}>0$ and $\sum_{j \in \Lambda} \varepsilon_{j} \leq 1$. Then for all $0<\beta<\beta_{c}$ $\left(\beta_{c}\right.$ defined below) the correlation function $G_{x y}(1.8)$ decays exponentially with the distance $|x-y|$. More precisely:

$$
\left\langle G_{x y}\right\rangle \leq C_{0}\left(\varepsilon_{x}^{-1}+\varepsilon_{y}^{-1}\right)\left[I_{\beta} e^{\beta\left(c_{d}-1\right)} c_{d}\right]^{|x-y|}
$$

where $c_{d}=2 d-1, C_{0}$ is a constant and

$$
I_{\beta}=\sqrt{\beta} \int_{-\infty}^{\infty} \frac{d t}{\sqrt{2 \pi}} e^{-\beta(\cosh t-1)} .
$$

Finally $\beta_{c}$ is defined by:

$$
I_{\beta_{c}} e^{\beta_{c}\left(c_{d}-1\right)} c_{d}=1
$$

Our estimates hold uniformly in the volume.

Remark 1 The integral $I_{\beta}$ satisfies $I_{\beta}<1 \forall \beta>0$ :

$$
I_{\beta}=\sqrt{\frac{\beta}{2 \pi}} \int_{-\infty}^{\infty} d t e^{-\beta(\cosh t-1)}<\sqrt{\frac{\beta}{2 \pi}} \int_{-\infty}^{\infty} d t \cosh (t / 2) e^{-\beta(\cosh t-1)}=1 .
$$

More precisely

$$
I_{\beta} \leq \begin{cases}\left(\ln \beta^{-1}\right) \sqrt{\beta} & \beta<0.15 \\ c e^{-\frac{1}{\beta}} & \beta>>1\end{cases}
$$

where $c>1$ is a constant.

Remark 2 The constraints $\varepsilon_{x}>0$ and $\varepsilon_{y}>0$ exclude the case of one pinning. Moreover $\sum_{j \in \Lambda} \varepsilon_{j} \leq 1$ implies $\varepsilon \leq \frac{1}{|\Lambda|}$ when $\epsilon_{j}=\varepsilon$ is constant. The case of one pinning is covered by Theorem 2 below. 
Main consequence For $d=1$ the critical beta is $\beta_{c}=\infty$ since

$$
I_{\beta} e^{\beta\left(c_{d}-1\right)} c_{d}=I_{\beta}<1 \quad \forall \beta>0 .
$$

Therefore the correlation function decays exponentially for all values of $\beta$. On the other hand for $d>1$ we obtain localization only for small $\beta$ since $\beta_{c}<(2 d-1)^{-2}<1$.

Theorem 2 Let $\varepsilon_{0}=O(1)$ and $\varepsilon_{j}=0 \forall j \neq 0$. Then for all $0<\beta<\beta_{c}$ $\left(\beta_{c}\right.$ defined below), the field $t_{x}$ wants to be as negative as $-|x|$. More precisely $\left\langle\mathcal{O}_{x}\right\rangle$ (defined in (1.9)) decays exponentially with the distance $|x-y|$

$$
\left\langle\mathcal{O}_{x}\right\rangle \leq C_{0}\left[I_{\beta} e^{\beta\left(c_{d}-1\right)} c_{d}\right]^{|x|},
$$

where $c_{d}$ and $I_{\beta}$ are defined in Theorem 1 above and $C_{0}$ is a constant. Finally $\beta_{c}$ is defined by:

$$
I_{\beta_{c}} e^{\beta_{c}\left(c_{d}-1\right)} c_{d}=1 .
$$

Our estimates hold uniformly in the volume.

Main consequence. For $d=1$ the critical beta is $\beta_{c}=\infty$ since

$$
I_{\beta} e^{\beta\left(c_{d}-1\right)} c_{d}=I_{\beta}<1 \quad \forall \beta>0 .
$$

Therefore $\left\langle\mathcal{O}_{x}\right\rangle$ decays exponentially for all values of $\beta$. On the other hand for $d>1$ the result holds only for small $\beta$ since $\beta_{c}<(2 d-1)^{-2}<1$.

Acknowledgments. It is our pleasure to thank A. Abdesselam for discussions and suggestions related to this paper. A special thanks to M. Zirnbauer who explained the model to us and shared his many insights.

\section{Proof of Theorem 1}

We want to estimate

$$
\left\langle G_{x y}\right\rangle=\int d \mu_{\Lambda}^{\varepsilon}(t) G_{x y}=\int \prod_{j \in \Lambda} \frac{d t_{j}}{\sqrt{2 \pi}} e^{-F_{\Lambda}(\nabla t)} e^{-M_{\Lambda}^{\varepsilon}(t)} \times \sqrt{\operatorname{det} D_{\Lambda}^{\varepsilon}(t)} D_{x y}^{-1} .
$$

The proof in done in four steps. 
Step 1. We mix the observable $G_{x y}$ and a piece of the probability measure namely $\sqrt{\operatorname{det} D}$. The key identity is

$$
\sqrt{\operatorname{det} D_{\Lambda}^{\varepsilon}(t)} D_{x y}^{-1}=\sqrt{D_{x y}^{-1}} \sqrt{D_{x y}^{-1} \operatorname{det} D_{\Lambda}^{\varepsilon}(t)}
$$

(remember that $D_{x y}^{-1}>0$ ). The first term is bounded by

$$
D_{x y}^{-1} \leq\left(\frac{1}{\varepsilon_{x} e^{-t_{x}}}+\frac{1}{\varepsilon_{y} e^{-t_{y}}}\right) \text {. }
$$

This is proved in Lemma 1, Inserting this in (2.1) we have

$$
\left\langle G_{x y}\right\rangle \leq \int d \nu_{\Lambda}^{\varepsilon}(t) \sqrt{\left[D_{x y}^{-1} \operatorname{det} D_{\Lambda}^{\varepsilon}(t)\right]}\left(\frac{e^{t_{x} / 2}}{\sqrt{\varepsilon_{x}}}+\frac{e^{t_{y} / 2}}{\sqrt{\varepsilon_{y}}}\right)
$$

where

$$
d \nu_{\Lambda}^{\varepsilon}(t)=\prod_{j \in \Lambda} \frac{d t_{j}}{\sqrt{2 \pi}} e^{-F_{\Lambda}(\nabla t)} e^{-M_{\Lambda}^{\varepsilon}(t)} .
$$

Unlike the measure $d \mu_{\Lambda}^{\varepsilon}$ given by (1.1), this measure is no longer normalized to 1 .

Step 2. We need to extract some decay in the distance $|x-y|$. This is hidden in $D_{x y}^{-1} \operatorname{det} D$. By some combinatorial arguments (the proof is given in Lemma 2 ) we can write

$$
\left[D_{x y}^{-1} \operatorname{det} D_{\Lambda}^{\varepsilon}(t)\right]=\sum_{\gamma_{x y}} \beta^{|\gamma|} \operatorname{det} D_{\Lambda_{\gamma}^{c}}^{\tilde{\varepsilon}^{c}}
$$

where the sum ranges over non self intersecting paths $\gamma$ made of nearest neighbor pairs in $\Lambda$ starting at $x$ and ending at $y$. Let $|\gamma|$, denote the length of $\gamma$ and let $\Lambda_{\gamma}$ be the corresponding set of lattice points and set $\Lambda_{\gamma}^{c}=\Lambda \backslash \Lambda_{\gamma}$. Finally $D_{\Lambda_{\gamma}^{c}}^{\tilde{\varepsilon}}$ is the matrix one obtains by deleting the rows and columns corresponding to the lattice points $j \in \Lambda_{\gamma}$. It is is exactly like the matrix $D_{\Lambda}^{\varepsilon}$, but defined on the complement of $\gamma, \Lambda_{\gamma}^{c}$ and with modified masses:

$$
\begin{aligned}
& D_{i j}^{\tilde{\varepsilon}}=0 \quad|i-j|>1 \\
& D_{i j}^{\tilde{\varepsilon}}=-\beta \quad|i-j|=1 \\
& D_{i i}^{\tilde{\varepsilon}}=\beta\left[2 d+\tilde{V}_{i}\right]+\tilde{\varepsilon}_{i} e^{t_{i}} \quad i=j,
\end{aligned}
$$


where $i, j \in \Lambda_{\gamma}^{c}$ and

$$
\begin{aligned}
& \tilde{V}_{i}=\sum_{k \in \Lambda_{\gamma}^{c},(k i)}\left[e^{t_{k}-t_{i}}-1\right] \\
& \tilde{\varepsilon}_{i}=\varepsilon_{i}+\beta \sum_{k \in \Lambda_{\gamma},(k i)} e^{t_{k}} .
\end{aligned}
$$

By combining (2.4) and (2.6) we have

$$
\left\langle G_{x y}\right\rangle \leq \sum_{\gamma_{x y}} \beta^{|\gamma| / 2} \int d \nu_{\Lambda}^{\varepsilon}(t)\left(\frac{e^{t_{x} / 2}}{\sqrt{\varepsilon_{x}}}+\frac{e^{t_{y} / 2}}{\sqrt{\varepsilon_{y}}}\right) \sqrt{\operatorname{det} D_{\Lambda_{\gamma}^{\tilde{\varepsilon}}}^{c}} .
$$

Step 3. The measure $d \nu_{\Lambda}^{\varepsilon}(t)$ defined in (2.5) can be factored as a measure on $\Lambda_{\gamma}$ times a measure on the complement set $\Lambda_{\gamma}^{c}$

$$
d \nu_{\Lambda}^{\varepsilon}(t)=d \nu_{\Lambda_{\gamma}}^{\varepsilon}(t) d \nu_{\Lambda_{\gamma}^{c}}^{\varepsilon}(t) e^{-F_{\partial \gamma}(\nabla t)},
$$

where

$$
F_{\partial \gamma}(\nabla t)=\sum_{(j, k), k \in \Lambda_{\gamma}, j \notin \Lambda_{\gamma}} \beta\left(\cosh \left(t_{j}-t_{k}\right)-1\right)
$$

describes the interaction between $\Lambda_{\gamma}$ and $\Lambda_{\gamma}^{c}$. Then the integral in (2.10) can be written as

$$
\int d \nu_{\Lambda}^{\varepsilon}(t)\left(\frac{e^{t_{x} / 2}}{\sqrt{\varepsilon_{x}}}+\frac{e^{t_{y} / 2}}{\sqrt{\varepsilon_{y}}}\right) \sqrt{\operatorname{det} D_{\Lambda_{\gamma}^{c}}^{\tilde{\varepsilon}_{c}}}=\int d \nu_{\Lambda_{\gamma}}^{\varepsilon}(t)\left(\frac{e^{t_{x} / 2}}{\sqrt{\varepsilon_{x}}}+\frac{e^{t_{y} / 2}}{\sqrt{\varepsilon_{y}}}\right) Z_{\Lambda_{\gamma}^{c}}^{\gamma}\left(t_{\gamma}\right),
$$

where we defined

$$
\begin{aligned}
Z_{\Lambda_{\gamma}^{c}}^{\gamma}\left(t_{\gamma}\right) & =\int d \nu_{\Lambda_{\gamma}^{c}}^{\varepsilon}(t) \sqrt{\operatorname{det} D_{\Lambda_{\gamma}^{c}}^{\tilde{\varepsilon}}} e^{-F_{\partial \gamma}(\nabla t)} \\
& =\int \prod_{j \in \Lambda_{\gamma}^{c}} \frac{d t_{j}}{\sqrt{2 \pi}} e^{-F_{\Lambda_{\gamma}^{c}}(\nabla t)} e^{-M_{\Lambda_{\gamma}^{c}}^{\varepsilon}(t)} \sqrt{\operatorname{det} D_{\Lambda_{\gamma}^{\tilde{\varepsilon}}}^{\tilde{\varepsilon}^{c}}} e^{-F_{\partial \gamma}(\nabla t)} .
\end{aligned}
$$

Note that $Z_{\Lambda_{\gamma}^{c}}^{\gamma}\left(t_{\gamma}\right)$ is still a function of the $t$ variables along the path $\left\{t_{k}\right\}_{k \in \Lambda_{\gamma}}$ (they are not integrated). Now $Z_{\Lambda_{\gamma}^{c}}^{\gamma}\left(t_{\gamma}\right)$ is almost equal to the partition function

$$
\begin{aligned}
1=Z_{\Lambda_{\gamma}^{c}}^{\tilde{\varepsilon}} & =\int d \mu_{\Lambda_{\gamma}^{c}}^{\tilde{\varepsilon}}(t)=\int d \nu_{\Lambda_{\gamma}^{c}}^{\tilde{\varepsilon}}(t) \sqrt{\operatorname{det} D_{\Lambda_{\gamma}^{c}}^{\tilde{\varepsilon}^{c}}} \\
& =\int \prod_{j \in \Lambda_{\gamma}^{c}} \frac{d t_{j}}{\sqrt{2 \pi}} e^{-F_{\Lambda_{\gamma}^{c}}(\nabla t)} e^{-M_{\Lambda_{\gamma}^{c}}^{\tilde{\varepsilon}_{c}}(t)} \sqrt{\operatorname{det} D_{\Lambda_{\gamma}^{\tilde{\varepsilon}}}^{\tilde{\varepsilon}}},
\end{aligned}
$$


where $Z_{\Lambda_{\gamma}^{c}}^{\tilde{\varepsilon}_{c}^{c}}=1$ by supersymmetry (see (1.7) $)$. Comparing (2.14) and $(\underline{2.15})$ we see that there are two main differences:

- the mass term $M_{\Lambda_{\gamma}^{c}}^{\varepsilon}$ in (2.14) depends on $\varepsilon$ instead of $\tilde{\varepsilon}$ and is smaller than what it should be

$$
M_{\Lambda_{\gamma}^{c}}^{\varepsilon} \leq M_{\Lambda_{\gamma}^{c}}^{\tilde{\varepsilon}}
$$

since $M^{\tilde{\varepsilon}}$ contains additional mass terms;

- the exponent in (2.14) contains the additional factor $-F_{\partial \gamma}(\nabla t)$ coming from the kinetic interaction between points on $\Lambda_{\gamma}$ and points on $\Lambda_{\gamma}^{c}$.

This last term is helping us since it makes the integral smaller. We will use it to recover the missing mass. This is done in Lemma 3 below. The key ingredient is a global translation on the $t$ variables. The result is

$$
\left\langle G_{x y}\right\rangle \leq e^{1} \sum_{\gamma_{x y}} \beta^{|\gamma| / 2} e^{\beta|\partial \gamma|} \int d \nu_{\Lambda_{\gamma}}^{\varepsilon}(t)\left(\frac{e^{t_{x} / 2}}{\sqrt{\varepsilon_{x}}}+\frac{e^{t_{y} / 2}}{\sqrt{\varepsilon_{y}}}\right)
$$

where $|\partial \gamma| \leq(2 d-2)|\gamma|+2$ is the number of points inside $\Lambda_{\gamma}^{c}$ on the boundary with $\Lambda_{\gamma}$.

Step 4. We are left with an integral along the path $\gamma$. The integral in (2.16) is bounded by

$$
\int d \nu_{\Lambda_{\gamma}}^{\varepsilon}(t)\left(\frac{e^{t_{x} / 2}}{\sqrt{\varepsilon_{x}}}+\frac{e^{t_{y} / 2}}{\sqrt{\varepsilon_{y}}}\right) \leq\left(\frac{I_{1}^{x}}{\sqrt{\varepsilon_{x}}}+\frac{I_{1}^{y}}{\sqrt{\varepsilon_{y}}}\right) I_{2}^{|\gamma|},
$$

where

$$
\begin{aligned}
& I_{1}^{x}=\int_{-\infty}^{\infty} \frac{d t}{\sqrt{2 \pi}} e^{t / 2} e^{-\varepsilon_{x}(\cosh t-1)}=\frac{1}{\sqrt{\varepsilon_{x}}} \\
& I_{2}=\int_{-\infty}^{\infty} \frac{d t}{\sqrt{2 \pi}} e^{-\beta(\cosh t-1)}=\frac{1}{\beta^{1 / 2}} I_{\beta}
\end{aligned}
$$


where $I_{\beta}$ was defined in (2.17). In the same way $I_{1}^{y}=1 / \sqrt{\varepsilon_{y}}$. Inserting all this we have

$$
\begin{aligned}
\left\langle G_{x y}\right\rangle & \leq e^{1}\left(\frac{1}{\varepsilon_{x}}+\frac{1}{\varepsilon_{y}}\right) \sum_{\gamma_{x y}} e^{\beta|\partial \gamma|}\left(I_{\beta}\right)^{|\gamma|} \\
& \leq 2 e^{1+2 \beta}\left(\frac{1}{\varepsilon_{x}}+\frac{1}{\varepsilon_{y}}\right) \sum_{n \geq|x-y|}\left(e^{\beta(2 d-2)} I_{\beta}\right)^{n} c_{d}^{n} \\
& \leq C_{0}\left(\frac{1}{\varepsilon_{x}}+\frac{1}{\varepsilon_{y}}\right)\left[e^{\beta(2 d-2)} I_{\beta} c_{d}\right]^{|x-y|}
\end{aligned}
$$

where $c_{d}=(2 d-1), C_{0}$ is a constant and the second inequality holds since the number of self-avoiding walks made of $n$ steps is bounded by $2 d(2 d-1)^{n}<2 c_{d}^{n}$ and $|\partial \gamma| \leq(2 d-2)|\gamma|+2$. Finally the sum over $n$ is convergent since $\left(e^{\beta(2 d-2)} I_{\beta} c_{d}\right)<1$.

This concludes the proof of Theorem 1 .

\subsection{The lemmas}

Lemma 1 The following inequality holds:

$$
D_{x y}^{-1} \leq \frac{e^{t_{x}}}{\varepsilon_{x}}+\frac{e^{t_{y}}}{\varepsilon_{y}}
$$

Proof By Cauchy-Schwartz inequality

$$
D_{x y}^{-1} \leq \sqrt{D_{x x}^{-1}} \sqrt{D_{y y}^{-1}} \leq D_{x x}^{-1}+D_{y y}^{-1} .
$$

Since $(f, D f) \geq \sum_{j} \varepsilon_{j} e^{-t_{j}} f_{j}^{2}$ for any $f_{j} \in \mathbb{R}$ we have

$$
D_{x x}^{-1} \leq \frac{1}{\varepsilon_{x} e^{-t_{x}}}
$$

Hence the result.

Lemma 2 For any invertible matrix $M$ on $\Lambda$ we have the following identity:

$$
\left[M_{x y}^{-1} \operatorname{det} M\right]=\sum_{\gamma_{x y}=\left(j_{1}, \ldots j_{m}\right)}\left[\left(-M_{x j_{1}}\right)\left(-M_{j_{1} j_{2}}\right) \cdots\left(-M_{j_{m} y}\right)\right] \operatorname{det}_{\Lambda_{\gamma}^{c}} M
$$

where $\gamma$ is any non self intersecting path starting at $x$ and ending at $y$. 
Proof This is a classical formula arising from the fact that every permutation can be decomposed as a product of cycles. One may derive it using the representation of a determinant as a sum over a gas of disjoint non self intersecting closed paths:

$$
\operatorname{det} M=\sum_{L_{1}, \ldots L_{p}} \mathcal{A}\left(L_{1}\right) \cdots \mathcal{A}\left(L_{p}\right)
$$

where a loop $L=\left(j_{1}, \ldots, j_{m}\right)$ is an ordered set of $m$ distinct points and

$$
\mathcal{A}(L)= \begin{cases}-\left[\left(-M_{j_{1} j_{2}}\right)\left(-M_{j_{2} j_{3}}\right) \cdots\left(-M_{j_{m} j_{1}}\right)\right] & m>1 \\ M_{j_{1} j_{1}} & m=1\end{cases}
$$

The sign $(-1)^{m-1}$ is the number of pairs inside the loop that need to be exchanged in order to recover the trivial permutation. Now since $\left[M_{x y}^{-1} \operatorname{det} M\right]=$ $\frac{\partial}{\partial M_{y x}} \operatorname{det} M$, the derivation selects only loops that contain the pair $y x$. The corresponding matrix element disappears and the loop becomes a path from $x$ to $y$. The sign -1 from $-M_{y x}$ cancels the global -1 in front of the product.

Lemma 3 For any configuration of $\left\{t_{k} \mid k \in \Lambda_{\gamma}\right\}$, the conditioned partition function $Z_{\Lambda_{\gamma}^{c}}^{\gamma}\left(t_{\gamma}\right)$ given by (2.14) is bounded by

$$
Z_{\Lambda_{\gamma}^{c}}^{\gamma}\left(t_{\gamma}\right) \leq e^{\beta \sum_{k \in \Lambda_{\gamma}} d_{k}^{\gamma}\left(1-e^{t_{k}-t^{*}}\right)} e^{\sum_{j \in \Lambda_{\gamma}^{c}} \varepsilon_{j}\left(1-e^{-t^{*}}\right)} \leq e^{\beta|\partial \gamma|} e^{\varepsilon\left|\Lambda_{\gamma}^{c}\right|}
$$

where $t^{*}$ is any real number satisfying

$$
t^{*} \geq 0, \quad \text { and } \quad t^{*} \geq t_{k} \quad \forall k \in \Lambda_{\gamma},
$$

and $d_{k}^{\gamma}$ is the number of points nearest neighbor to $k$ that do not belong to $\Lambda_{\gamma}$ :

$$
d_{k}^{\gamma}=\#\left\{j \notin \Lambda_{\gamma}|| j-k \mid=1\right\} .
$$

Proof Before doing any bound we perform a global translation inside the integral:

$$
t_{j} \rightarrow t_{j}+t^{*} \quad \forall j \in \Lambda_{\gamma}^{c} .
$$


Then inside the exponential we have:

$$
\begin{aligned}
F_{\Lambda_{\gamma}^{c}}(\nabla t) & \mapsto F_{\Lambda_{\gamma}^{c}}(\nabla t) \\
M_{\Lambda_{\gamma}^{c}}^{\varepsilon}(t) & \mapsto M_{\Lambda_{\gamma}^{c}}^{\varepsilon}\left(t+t^{*}\right) \\
F_{\partial \gamma}(\nabla t) & \mapsto F_{\partial \gamma}\left(\nabla t+t^{*}\right)=\sum_{(j, k), k \in \Lambda_{\gamma}, j \in \Lambda_{\gamma}^{c}} \beta\left(\cosh \left(t_{j}+t^{*}-t_{k}\right)-1\right) .
\end{aligned}
$$

In the numerator we have

$$
\left[\operatorname{det} D_{\Lambda_{\gamma}^{c}}^{\tilde{\varepsilon}^{c}}\right] \mapsto\left[\operatorname{det} D_{\Lambda_{\gamma}^{c}}^{\tilde{\varepsilon^{-t^{*}}}}\right]
$$

so the only effect of the translation is to modify the mass $\tilde{\varepsilon}_{j}$ defined in (2.9) at each lattice point. After the translation

$$
\begin{aligned}
& Z_{\Lambda_{\gamma}^{c}}^{\gamma}=\int\left[\prod_{j \in \Lambda_{\gamma}^{c}} \frac{d t_{j}}{\sqrt{2 \pi}}\right] e^{-F_{\Lambda_{\gamma}^{c}}(\nabla t)} \sqrt{\operatorname{det} D_{\Lambda_{\gamma}^{c}}^{\tilde{\varepsilon} e^{-t^{*}}}} e^{-M_{\Lambda_{\gamma}^{c}}^{\varepsilon}\left(t+t^{*}\right)} e^{-F_{\partial \gamma}\left(\nabla t+t^{*}\right)} \\
& =\int\left[\prod_{j \in \Lambda_{\gamma}^{c}} \frac{d t_{j}}{\sqrt{2 \pi}}\right] e^{-F_{\Lambda c}^{c}(\nabla t)} e^{-M_{\Lambda_{\gamma}^{c}}^{\tilde{\varepsilon}} e^{-t^{*}}(t)} \sqrt{\operatorname{det} D_{\Lambda_{\gamma}^{c}}^{\tilde{\varepsilon} e^{-t^{*}}}} e^{M_{\Lambda_{\gamma}^{c}}^{\tilde{\varepsilon}^{-t^{*}}}(t)-M_{\Lambda_{\gamma}^{c}}^{\varepsilon}\left(t+t^{*}\right)-F_{\partial \gamma}\left(\nabla t+t^{*}\right)}
\end{aligned}
$$

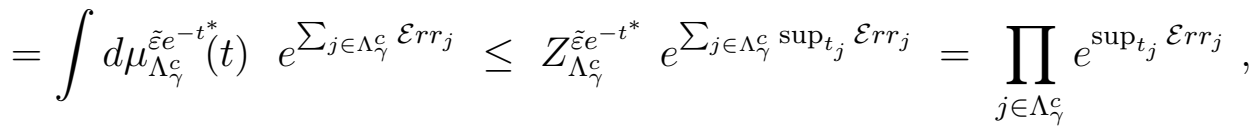

where we used $Z_{\Lambda_{\gamma}^{c}}^{\tilde{\varepsilon} e^{-t^{*}}}=1$ (see (1.7)) and we defined

$$
\begin{gathered}
\sum_{j \in \Lambda_{\gamma}^{c}} \mathcal{E} r r_{j}=\left[M_{\Lambda_{\gamma}^{c}}^{\tilde{\varepsilon} e^{-t^{*}}}(t)-M_{\Lambda_{\gamma}^{c}}^{\varepsilon}\left(t+t^{*}\right)-F_{\partial \gamma}\left(\nabla t+t^{*}\right)\right] \\
\mathcal{E} r r_{j}=\tilde{\varepsilon}_{j} e^{-t^{*}}\left(\cosh t_{j}-1\right)-\varepsilon_{j}\left(\cosh \left(t_{j}+t^{*}\right)-1\right)-\sum_{k \in \Lambda_{\gamma},(j, k)} \beta\left(\cosh \left(t_{j}+t^{*}-t_{k}\right)-1\right) .
\end{gathered}
$$

To conclude we shall prove that the right hand side of (2.29) is bounded by the rhs of (2.23):

$$
\prod_{j \in \Lambda_{\gamma}^{c}} e^{\sup _{t_{j}} \mathcal{E} r r_{j}} \leq e^{\beta \sum_{k \in \Lambda_{\gamma}} d_{k}^{\gamma}\left(1-e^{t_{k}-t^{*}}\right)} e^{\sum_{j \in \Lambda_{\gamma}^{c}} \varepsilon_{j}\left(1-e^{-t^{*}}\right)} .
$$

We distinguish two cases. 
Case 1. When $j$ is far from the path $\gamma$ that is $|j-k|>1$ for all $k \in \Lambda_{\gamma}$ then $\tilde{\varepsilon}_{j}=\varepsilon_{j}$ and we have

$$
\begin{aligned}
\mathcal{E} r r_{j} & =\varepsilon_{j}\left[e^{-t^{*}}\left(\cosh t_{j}-1\right)-\left(\cosh \left(t_{j}+t^{*}\right)-1\right)\right] \\
& =\varepsilon_{j}\left[e^{t_{j}} \sinh \left(-t^{*}\right)+1-e^{-t^{*}}\right] \leq \varepsilon_{j}\left(1-e^{-t^{*}}\right),
\end{aligned}
$$

where the last inequality holds for $t^{*} \geq 0$.

Case 2. When $j$ is near to the path $\gamma$ that is $|j-k|=1$ for some $k \in \Lambda_{\gamma}$ then $\tilde{\varepsilon}_{j}=\varepsilon_{j}+\sum_{k \in \Lambda_{\gamma},(j, k)} \beta e^{t_{k}}$ and we have

$$
\begin{aligned}
\mathcal{E r r}_{j}=\varepsilon_{j} & {\left[e^{-t^{*}}\left(\cosh t_{j}-1\right)-\left(\cosh \left(t_{j}+t^{*}\right)-1\right)\right] } \\
& +\beta \sum_{k \in \Lambda_{\gamma},(j, k)}\left[e^{t_{k}-t^{*}}\left(\cosh t_{j}-1\right)-\left(\cosh \left(t_{j}+t^{*}-t_{k}\right)-1\right)\right] \\
= & \varepsilon_{j}\left[e^{t_{j}} \sinh \left(-t^{*}\right)+1-e^{-t^{*}}\right]+\beta \sum_{k \in \Lambda_{\gamma},(j, k)}\left[e^{t_{j}} \sinh \left(t_{k}-t^{*}\right)+1-e^{t_{k}-t^{*}}\right] \\
\leq & \varepsilon_{j}\left(1-e^{-t^{*}}\right)+\beta \sum_{k \in \Lambda_{\gamma},(j, k)}\left(1-e^{t_{k}-t^{*}}\right)
\end{aligned}
$$

where the last inequality holds if $t^{*} \geq 0$ and $t^{*} \geq t_{k} \forall k \in \Lambda_{\gamma}$.

Finally

$$
\begin{aligned}
\sum_{j \in \Lambda_{\gamma}^{c}} \mathcal{E} r r_{j} & \leq \sum_{j \in \Lambda_{\gamma}^{c}}\left[\varepsilon_{j}\left(1-e^{-t^{*}}\right)+\beta \sum_{k \in \Lambda_{\gamma},(j, k)}\left(1-e^{t_{k}-t^{*}}\right)\right] \\
& \leq \beta \sum_{k \in \Lambda_{\gamma}} d_{k}^{\gamma}\left(1-e^{t_{k}-t^{*}}\right)+\sum_{j \in \Lambda_{\gamma}^{c}} \varepsilon_{j}\left(1-e^{-t^{*}}\right) .
\end{aligned}
$$

This concludes the proof of the lemma.

\section{Proof of Theorem 2}

The proof of Theorem 2 is almost identical to that of Theorem 1 . This time there is no term $D_{x y}^{-1}$ ensuring we can extract a path $\gamma$ connecting $x$ to $y$. On the other hand, since there is a pinning only at one position the matrixtree theorem (see [1] for a simple proof and many references) applied to the "diffusion" representation $A$ given in (1.5) of the matrix $D$ gives

$$
\operatorname{det} A=\varepsilon_{0} e^{t_{0}} \sum_{T} \prod_{j j^{\prime} \in T}\left(-A_{j j^{\prime}}\right)=\varepsilon_{0} e^{t_{0}} \sum_{T} \prod_{\left(j j^{\prime}\right) \in T}\left[\beta e^{t_{j}+t_{j^{\prime}}}\right],
$$


where the sum is over the spanning trees on $\Lambda$ made of nearest neighbor pairs (since $A_{j j^{\prime}}=0$ when $\left|j-j^{\prime}\right|>1$ ). Therefore each term in the sum contains a path $\gamma$ from 0 to $x$. Actually using (3.1) it is easy to see that

$$
A_{0 x}^{-1} \operatorname{det} A=\frac{1}{\varepsilon_{0} e^{t_{0}}} \operatorname{det} A
$$

for all $x \in \Lambda, x \neq 0$. Therefore $\varepsilon_{0} e^{t_{0}} A_{0 x}^{-1}=1$ and

$$
\operatorname{det} D=\varepsilon_{0} e^{t_{0}} A_{0 x}^{-1} \operatorname{det} D=\varepsilon_{0} e^{-t_{x}} D_{0 x}^{-1} \operatorname{det} D=\varepsilon_{0} e^{-t_{x}} \sum_{\gamma_{0 x}} \beta^{|\gamma|} \operatorname{det}_{\Lambda_{\gamma}^{c}} D,
$$

where $A_{0 x}^{-1}=e^{-t_{0}} D_{0 x}^{-1} e^{-t_{x}}$ and in the last term we applied Lemma 2. Inserting this result in (1.1) we have

$$
\begin{aligned}
& \left\langle\mathcal{O}_{x}\right\rangle=\int d \mu_{\Lambda}^{\varepsilon}(t) \mathcal{O}_{x}=\int d \nu_{\Lambda}^{\varepsilon}(t) \sqrt{\operatorname{det} D_{\Lambda}^{\varepsilon}} e^{t_{x} / 2} \\
& =\sqrt{\varepsilon_{0}} \int d \nu_{\Lambda}^{\varepsilon}(t) e^{t_{x} / 2} e^{-t_{x} / 2} \sqrt{\sum_{\gamma_{0 x}} \beta|\gamma| \operatorname{det} D_{\Lambda_{\gamma}^{c}}^{\tilde{\varepsilon}}} \\
& \leq \sqrt{\varepsilon_{0}} \sum_{\gamma_{0 x}} \beta^{|\gamma| / 2} \int d \nu_{\Lambda_{\gamma}}^{\varepsilon}(t) Z_{\Lambda_{\gamma}^{c}}^{\gamma}\left(t_{\gamma}\right) \leq \sqrt{\varepsilon_{0}} \sum_{\gamma_{0 x}} \beta^{|\gamma| / 2} \int d \nu_{\Lambda_{\gamma}}^{\varepsilon}(t) e^{\beta|\partial \gamma|} \\
& \leq \sum_{\gamma_{0 x}} e^{\beta|\partial \gamma|} I_{\varepsilon_{0}} I_{\beta}^{|\gamma|} \leq C_{0}\left(c_{d} e^{\beta\left(c_{d}-1\right)} I_{\beta}\right)^{|x|}
\end{aligned}
$$

where $d \nu_{\Lambda}^{\varepsilon}(t)$ was defined in (2.5),$Z_{\Lambda_{\gamma}^{c}}^{\gamma}\left(t_{\gamma}\right)$ in (2.14),$I_{\beta}$ in (1.13) and the same definition holds for $I_{\varepsilon}$. In the second line we $u$ sed $\operatorname{det}_{\Lambda_{\gamma}^{c}} D=\operatorname{det} D_{\Lambda_{\gamma}^{c}}^{\tilde{\varepsilon}}$ (see (2.7)). We used Lemma 3 eq. (2.23) to bound $Z_{\Lambda_{\gamma}^{c}}^{\gamma}\left(t_{\gamma}\right)$. Finally the last inequality holds since $\left(c_{d} e^{c_{d}-1} I_{\beta}\right)<1$. This concludes the proof of Theorem 2 .

\section{References}

[1] A. Abdesselam. The Grassmann-Berezin calculus and theorems of the matrix-tree type. Adv. in Appl. Math., 33(1):51-70, 2004.

[2] M. Disertori, T. Spencer, and M.R. Zirnbauer. Quasi-diffusion in a 3d supersymmetric hyperbolic sigma model. arXiv:0901.1652. 
[3] W. Drunk, D. Fuchs, and M.R. Zirnbauer. Migdal-Kadanoff renormalization of a nonlinear supervector model with hyperbolic symmetry. Ann. Physik, 1:134-150, 1992.

[4] T. Dupré. On the localization transition in three dimensions: Monte-Carlo simulation of a non-linear $\sigma$-model. Physical Review B, 54(18):2763-12774, 1996.

[5] K. B. Efetov. Supersymmetry and theory of disordered metals. Adv. Phys., 32:874-, 1983.

[6] K. B. Efetov. Supersymmetry in disorder and chaos. Cambridge University Press, Cambridge, 1997.

[7] F. Merkl and S.W.W. Rolles. Linearly edge-reinforced random walks, volume 48 of IMS Lecture Notes-Monograph Series, pages 66-77. 2006.

[8] F. Wegner. The mobility edge problem: continuous symmetry and a conjecture. Z. Phys. B, 35:207-210, 1979.

[9] F. Wegner and L. Schaefer. Disordered system with $n$ orbitals per site: Lagrange formulation, hyperbolic symmetry, and goldstone modes. $Z$. Phys. B, 38:113-126, 1980.

[10] M.R. Zirnbauer. Fourier analysis on a hyperbolic supermanifold with constant curvature. Commun. Math. Phys., 141:503-522, 1991. 